\title{
Expression and Function of RIG-I in Oral Keratinocytes and Fibroblasts
}

\author{
Kouji Ohta Akiko Fukui Hideo Shigeishi Yoko Ishida Hiromi Nishi Kei Tobiume \\ Masaaki Takechi Nobuyuki Kamata
}

Department of Oral and Maxillofacial Surgery, Division of Cervico-Gnathostmatology, Programs for Applied Biomedicine, Graduate School of Biomedical Sciences, Hiroshima University, 1-2-3 Kasumi, Minami-Ku, Hiroshima, Japan

\section{Key Words}

RIG-I • IFN- $\beta \cdot \mathrm{CXCL10} \cdot$ Oral keratinocytes $\bullet$ Oral fibroblasts

\begin{abstract}
Background: Innate immune response by oral mucosal cells may be the first line of host defense against viral infection. Retinoic acid-inducible gene-I (RIG-I) recognizes viral dsRNA in the cytoplasm, and RIG-I-mediated signaling regulates antiviral type I IFN, and inflammatory chemokine production. Here, we tested the hypothesis that oral mucosal cell participation in host defense against viral infection via RIG-I. Methods: RIG-I expression was detected in immortalized oral keratinocytes (RT7), oral fibroblasts (GT1) using and RT-PCR and immunohistochemistry. RT7 and GT1 were exposed to dsRNA virus mimic Poly I:C-LMW/ LyoVec (PLV). Expression of IFN- $\beta$ and CXCL10 via RIG-I was examined by Real-time RT-PCR and ELISA. Phosphorylation of IRF3 and STAT1 were detected by western blotting. Results: RT7 and GT1 constitutively expressed RIG-I in the cytoplasm. Furthermore, PLV increased IFN- $\beta$ and CXCL10 productions in both RT7 and GT1 via RIG-I concurrent with phosphorylation of IRF3 and STAT1. PLV-induced CXCL10 production was attenuated by neutralization of IFN- $\beta$ and blocking of IFN $-\alpha / \beta$ receptor (IFNAR), indicating primal IFN- $\beta$ production via the RIG-IIRF3 axis, which eventually induces CXCL10 production via the IFNAR -STAT1 axis. Conclusion: We propose that RIG-I in oral keratinocytes and fibroblasts may cumulatively develop hostdefense mechanisms against viral infection in oral mucosa.
\end{abstract}

Copyright $\odot 2014$ S. Karger AG, Basel

\section{Introduction}

The innate immune system constitutes the first line of host defense against invading viruses. Viral signature patterns including viral DNA and RNA are recognized by both endosomal membrane and cytoplasmic receptors in various cell types [1]. Toll-like receptors 
(TLRs) are transmembrane receptors located on the cell surface and endosomes that bind extracellular pathogens and set off a signaling cascade as innate immune responses [2]. Cytoplasmic pattern recognition receptors including DExH box family RNA helicase proteins such as retinoic acid-inducible gene-I (RIG-I) play distinct roles in sensing intracellular nucleic acid to trigger mitochondrial adaptor protein IFN- $\beta$ promoter stimulator 1 (IPS1)dependent innate immune signaling response [3]. RIG-I recognizes viral dsRNA in the cytoplasm, and RIG-I-mediated signaling regulates antivirus type I IFN, such as IFN- $\alpha / \beta$ and various chemokines that play critical roles in initiating ant-virus innate and modulating subsequent adapting immunity including migration of leucocytes at the site of infection [4].

Oral mucosa is thought to be constantly exposed to various pathogens, and serves as a barrier against microorganism invasion [5]. Oral keratinocytes and fibroblasts are the major cell types present in oral mucosa, thus they are considered to be major sources of early production of humoral factors such as cytokines and chemokines at the site of infection. Previously, we showed that oral keratinocytes and fibroblasts express various TLRs, which recognize extracellular microorganism components via pathogen-associated molecular patterns (PAMPS) [6]. However, it remains unknown whether oral keratinocytes and fibroblasts sense intracellular invader nucleic acid signatures via functional RIG-I.

CXCL10, a chemokine associated with type $1 \mathrm{~T}$ cell responses, regulates the migration of activated T cells through binding to the CXCR3 receptor [7]. We previously reported that oral keratinocytes and fibroblasts produce CXCL10, which is thought to attract immune cells to the site of oral inflammation [8]. The major function of CXCL10 in host defense against viral infection has been shown in CXCL10-/- mice, in which it impaired recruitment of effector $\mathrm{T}$ cells and NK cells to sites of infection/inflammation [9, 10].

We hypothesized that RIG-I in oral keratinocytes as well as fibroblasts recognizes viral infection, and participates following in the activated host defense system. In the present study, we examined RIG-I expression, and investigated the effects of RIG-I agonists on production of innate humoral factors, such as IFN- $\beta$ and CXCL10.

\section{Materials and Methods}

Reagents

Poly I:C/LMW/LeoVec, a complex of low molecular-weight poly I:C and the transfection reagent LyoVec as well as naked Poly I:C/LMW, and BX795, a TBK1 and IKKe inhibitor, were purchased from Invivogen (San Diego, CA, USA). The JAK/STAT inhibitor, AG490 was from Cayman Chemical Co (Michigan, USA). Antiserum against human RIG-I was purchased from GeneTex (CA, USA). The antibodies used for immunoblotting were anti-IRF3, anti-phospho-IRF3, anti-STAT1, and anti-phospho- STAT1 from Cell Signaling Technology (Danvers, MA), while secondary antibodies for labeling were HRP-conjugated antibody from GE Healthcare Bio-Sciences (Tokyo, JAPAN) and Alexa 488-conjugated rabbit Ig from Invitrogen (Carlsbad, CA, USA). Neutralizing antibodies for IFN- $\beta$ and the IFN- $\alpha / \beta$ receptor, and control rabbit IgG were purchased from R\&D Systems (Minneapolis, MI, USA).

\section{Cell lines}

The immortalized human oral keratinocyte cell line RT7 and human oral fibroblast cell line GT1, were established, as previously described $[11,12]$. Primary cultures of human keratinocytes and fibroblasts from oral mucosal biopsy samples from healthy volunteers were prepared as previously described [8]. Informed consent for acquisition was obtained according to a protocol approved by the Ethical Committee of Hiroshima University. Keratinocytes were cultured in Keratinocytes-SFM (Gibco BRL, Gaithersburg, MD, USA) including $25 \mu \mathrm{g} / \mathrm{mL}$ bovine pituitary extract, $0.05 \mathrm{ng} / \mathrm{mL}$ epidermal growth factor, $100 \mathrm{U} / \mathrm{mL}$ penicillin, and $100 \mu \mathrm{g} /$ $\mathrm{mL}$ streptomycin. Fibroblasts were cultured in Dulbecco's modified Eagle's medium (Sigma Chemical Co., St. Louis, MO, USA) containing 10\% fetal calf serum, $100 \mathrm{U} / \mathrm{mL}$ penicillin, and $100 \mu \mathrm{g} / \mathrm{mL}$ streptomycin.

Buccal mucosal cell collections from swabs

Buccal cells were collected as follows [13]. The samples were obtained from healthy subjects after obtaining informed consent for acquisition was obtained according to a protocol approved by the Ethical 
Committee of Hiroshima University. A cytologic brush was spun in the buccal mucosa for 10 seconds in a single direction, while constant pressure was applied to the mucosa. The brush was then withdrawn, immediately plunged into the RNA preservative buffer (Ambion, Austin, TX, USA) at room temperature, and then frozen for later use.

\section{Immunocytochemistry}

Cells were seeded into 8 well-chamber slide (Matsunami Glass, Osaka, Japan) and fixed in 4\% paraformaldehyde in PBS for 15 minutes, followed by permeabilization with $0.2 \%$ Triton X-100 in PBS for 5 minutes and incubation overnight with primary antibodies diluted in PBS containing 5\% BSA. Next, the cells were washed and incubated with diluted Alexa Fluor secondary antibody (Invitrogen) for $1 \mathrm{hr}$. Vectashield anti-fade medium containing DAPI (Vector Laboratories, Burlingame, CA, USA) was used to mount the cells. Fluorescent and phase contrast images were acquired using a BZ-9000 microscope (KEYENCE, Osaka, Japan).

\section{Small interfering RNA}

A stealth small interfering RNA (siRNA) for RIG-I, and negative control siRNA were purchased from Invitrogen. RT7 and GT1 were transiently transfected with various combinations of the siRNAs using Lipofectamine ${ }^{\circledR} 2000$ transfection reagent (Invitrogen), according to the manufacturer's recommendations.

RNA extraction, RT-PCR, and real-time PCR

The gene-specific oligonucleotide primers used for PCR analysis were as follows : RIG-I sense 5'-AGG AAA ACT GGC CCA AAA CT-3' and antisense 5'-TTT CCC CTT TTG TCC TTG TG-3', IFN- $\beta$ sense 5'-TGC TCT GGC ACA ACA GGT AG-3' and antisense 5'-GCT GCA GCT GCT TAA TCT CC-3', CXCL10 sense 5'- GCA GCT GAT TTG GTG ACC ATC ATT GG-3' and antisense 5'-TGC AAG CCA ATT TTG TCC ACG TGT TG-3'. TLR3 sense 5'-AAA TTG GGC AAG AAC TCA CAG G-3' and antisense 5'-GTG TTT CCA GAG CCG TGC TAA-3', MDA5 sense 5'-CTG CTG CAG AAA ACA ATG GA-3' and antisense 5'-TGC CCA TGT TGC TGT TAT GT-3', LGP-2 sense 5'-ATG TGA ACC CCA ACT TCT CG-3' and antisense 5'-CAA TGC TGC AGG AAG TCA AA-3'

Total RNA was prepared from the cell lines using an RNeasy total RNA isolation Kit (Qiagen, Hilden, Germany). One-step RT-PCR was performed using an RT-PCR High Plus System (Toyobo, Osaka, Japan) according to the manufacturer's instructions. Single-stranded cDNA for RT-PCR and a quantitative real-time PCR template were synthesized using a First Strand cDNA Synthesis Kit (Amersham Biosciences, Uppsala, Sweden). The RT-PCR conditions for RIG-I were $1 \times\left(95^{\circ} \mathrm{C}, 15\right.$ minutes $), 35 \times\left(95^{\circ} \mathrm{C}, 2\right.$ minutes; $55^{\circ} \mathrm{C}, 30$ seconds; $72^{\circ} \mathrm{C}, 1$ minute) and $1 \times\left(72^{\circ} \mathrm{C}, 7\right.$ minutes), and those for TLR3, MDA5, and LGP2 were $1 \times\left(95^{\circ} \mathrm{C}, 15\right.$ minutes $), 30 \times\left(95^{\circ} \mathrm{C}, 2\right.$ minutes; $55^{\circ} \mathrm{C}, 30$ seconds; $72^{\circ} \mathrm{C}, 1$ minute $)$ and $1 \times\left(72^{\circ} \mathrm{C}, 7\right.$ minutes $)$, while those for $\beta$-actin were $1 \times\left(95^{\circ} \mathrm{C}, 15\right.$ minutes $), 25 \times\left(95^{\circ} \mathrm{C}, 2\right.$ minutes; $55^{\circ} \mathrm{C}, 30$ seconds; $72^{\circ} \mathrm{C}, 1$ minute $)$, and $1 \times\left(72^{\circ} \mathrm{C}\right.$, 7 minutes). Quantitative real-time PCR was performed using SYBR-Green Master Mix (Applied Biosystems, Carlsbad, CA, USA) for 40 cycles at $95^{\circ} \mathrm{C}$ for 15 seconds and $60^{\circ} \mathrm{C}$ for 60 seconds. Quantitative PCR analysis was performed using a CFX Connect Real-Time PCR Detection System (Bio-Rad Laboratories, Hercules, CA, USA). Relative quantification of given mRNA levels among the samples was performed according to User Bulletin \#2 (Applied Biosystems), with the results shown as the mean \pm standard deviation from 3 independent experiments.

\section{Cytokine determination}

Cell culture supernatants were collected, and the concentration of IFN- $\beta$ and CXCL10 were measured using an IFN- $\beta$ ELISA kit (Fujirebio, Tokyo, JAPAN) and CXCL10 ELISA kit (R\&D Systems), respectively, as previously described [6].

\section{Western blotting}

Cells were harvested using a Mammalian Cell Lysis Kit (Sigma-Aldrich). Protein concentrations were determined using a protein assay kit (Bio-Rad Laboratories) and $25 \mu \mathrm{g}$ of protein from each sample was separated on $10 \%$ SDS-polyacrylamide gels, then transferred to polyvinylidene fluoride membranes (Amersham Biosciences). After incubation with the specific antibody, immunoblots were labeled with the HRP-conjugated secondary antibody and developed using an ECL Advance Western Blotting Detection Kit (GE Healthcare Life Sciences, Tokyo, Japan). Image data were analyzed with an LAS 4000 mini imaging system (Fuji Film, Tokyo, Japan). 
Fig. 1. Expression of RIG-I in oral keratinocytes and fibroblasts. (A)RIG-I mRNA expression in RT7, GT1, primary oral keratinocyts, fibroblasts cell lines, and buccal mucosal cells derived from swabs. Human buccal mucosal cells from 3 healthy volunteers (1 male, aged 40 years, 2 females, aged 29 , and 30 years, respectively.) were obtained as previously reported [13]. Total RNA was isolated from each cell line cultured to confluence then RT-PCR assays were performed for RIG-I and $\beta$-actin. (B) Expression of RIG-I protein in oral keratinocytes and fibroblasts. Green staining indicating RIG-I was observed in the cytoplasm of the cells.

\section{Statistical analysis}

Data were analyzed using Student's t-test or one-way analysis of variance (ANOVA), and the results are presented as the mean \pm standard deviation.

\section{Results}

Oral keratinocytes and fibroblasts expressed RIG-I

To investigate the potential contribution of oral mucosal non-immune cells to innate antiviral response via RIG-I, we first examined whether oral keratinocytes and fibroblasts express RIG-I. RT-PCR results showed that pure keratinocytes and fibroblasts in cultures constitutively expressed RIG-I mRNA, whereas buccal mucosal cells derived from swabs variously expressed RIG-I (Fig. 1A). RIG-I was detected in the cytoplasm of both RT7 and GT1 when analyzed by immunocytochemistry (Fig. 1B), indicating that RIG-I was functional in those cells. Since the primary cells showed limited proliferative activity, we subjected immortalized oral keratinocytes and fibroblasts to the following analyses.

\section{PLV induced IFN- $\beta$ and CXCL10 in oral mucosal cells via RIG-I}

We used poly I:C-LMW/LyoVec (PLV), a complex of low molecular weight poly I:C and the transfection reagent LyoVec as a potential RIG-I ligand [14]. IFN- $\beta$, is produced by nonimmune cells, such as fibroblasts infected with a virus, is a Type1 cytokine. Therefore, we focused on IFN- $\beta$ as Type I IFN [15]. When exposed to PLV for $12 \mathrm{hrs}$, mRNA levels of IFN- $\beta$ showed significant increases in both RT7 and GT1 (Fig. 2A). We also found that CXCL10 mRNA was dramatically induced by PLV in a dose-dependent manner (Fig. 2B). To examine whether intercellular response predominantly functioned in transcribed dsRNA, we compared the effects of naked low molecular weight poly I:C (Poly I:C/LMW) and PLV, and found that PLV increased CXCL10 in comparison with Poly:IC LMW in both cells (Fig. 2C).

To determine whether RIG-I was actually associated with the increase of IFN- $\beta$ and CXCL10 in response to the possible ligand PLV, we performed siRNA-mediated knockdown 
Fig. 2. Effects of induction of PLV on IFN- $\beta$ and CXCL10. (A) (B) Effects of PLV on IFN- $\beta$ and CXCL10 mRNA in RT7 and GT1. Cells were exposed to various concentrations of PLV for 12 hrs. Data are shown as the mean \pm standard deviation of 3 independent experiments. *Significant increase as compared with non-treated cells (Student's $t$-test: $\mathrm{p}<0.05$ ). (C) Effects of Poly I:C/LMW and PLV on CXCL10 in RT7 and GT1. Cells were exposed to various concentrations of Poly I:C/LMW and PLV for $12 \mathrm{hrs}$. Data are shown as the mean \pm standard deviation of 3 independent experiments. * Significant increase as compared with non-treated cells (Student's t-test: $\mathrm{p}<0.05)$. \#Significant increase as compared with Poly I:C/LMW (Student's t-test: $\mathrm{p}<0.05$ ).
A)
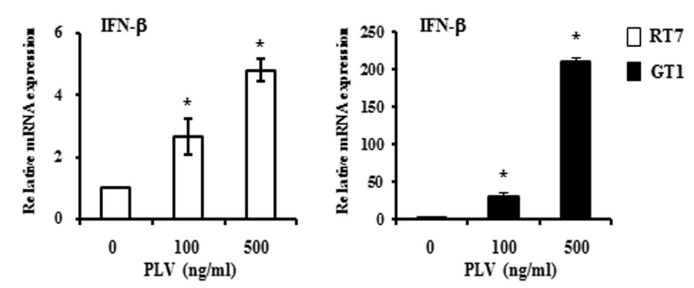

B)
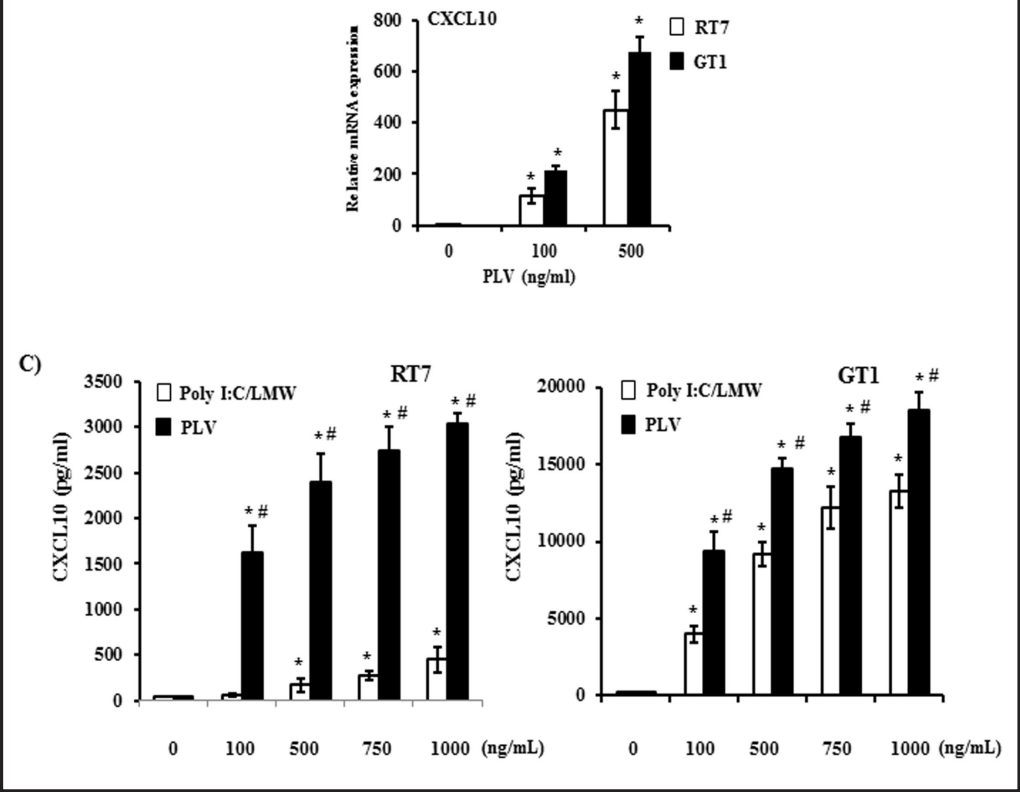

of RIG-I. The specific SiRNA for RIG-I decreased the mRNA expression of RIG-I, but not that of the other RNA sensor genes, such as TLR3, MDA5, and LGP3 (Fig. 3A). The production levels of both IFN- $\beta$ and CXCL10 induced by PLV were specifically attenuated by siRNAs to RIG-I designed differently, indicating that RIG-I contributed to the PLV induced production of IFN- $\beta$ and CXCL10 (Fig. 3 B).

\section{PLV activated IRF3 and STAT1 in oral mucosal cells}

To elucidate which signaling pathway functioned in the induction of IFN- $\beta$ and CXCL10, we evaluated the site-specific phosphorylation of IRF-3 and STAT1, which are transcriptional factors that activate transcription for IFN- $\beta$ and CXCL10, respectively $[16,17]$. Exposure to PLV markedly induced phosphorylation of IRF3 and STAT1 in both RT7 and GT1 (Fig. 4A). Since site-specific phosphorylation of IRF3 is catalyzed by TBK1 and IKKe $[18,19]$, the specific inhibitor of these kinases BX795 was tested. When exposed to PLV with increasing amounts of BX795, both RT7 and GT1 clearly showed a reduced level of PLV-dependent IFN- $\beta$ and CXCL10 induction in a dose-dependent manner (Fig. 4B,C). Similarly, the JAK inhibitor AG490, which prevents site-specific phosphorylation of STAT1 by this kinase, dramatically decreased PLV-induced CXCL10 production in both RT7 and GT1 in a dosedependent manner (Fig. 4C). On the other hand, AG490 did not affect PLV-induced IFN- $\beta$ in either cell type indicating that STAT1 was not responsible for PLV-induced INF- $\beta$ production (Fig. 4B). Knockdown of RIG-I in RT7 and GT1 confirmed that the phosphorylation of STAT1 in response to exposure to PLV, was dependent on RIG-I (Fig. 4D).

PLV induced CXCL10 in oral mucosal cells via RIG-I-dependent IFN- $\beta$ production

Although the best known activator to lead CXCL10 production is type II interferon, which activate STAT1 homodimer, type I interferon also has an ability to lead the activates 
Fig. 3.Effects of induction of PLV via RIG-I on IFN- $\beta$ and CXCL10. (A) Effects of siRNA for RIG-I on mRNA expression of RIG-I and expression of other RNA sensor genes in RT7 and GT1. Cells were transiently transfected with the indicated combinations of siRNAs for RIG-I, and a transfection reagent for $48 \mathrm{hrs}$. Total RNA was isolated from each cell line after culturing to confluence then RT-PCR assays were performed for RIGI, other RNA sensor genes, and $\beta$-actin. (B) Effects of siRNA for RIG-I on PLV-induced IFN- $\beta$ and CXCL10 in RT7 and GT1. Cells were transiently transfected with the indicated combinations of the siRNAs and transfection reagent for $48 \mathrm{hrs}$, then exposed to PLV (500 ng/mL) for $24 \mathrm{hrs,} \mathrm{after}$ which the levels of IFN- $\beta$ and CXCL10 in culture supernatant were measured by ELISA.

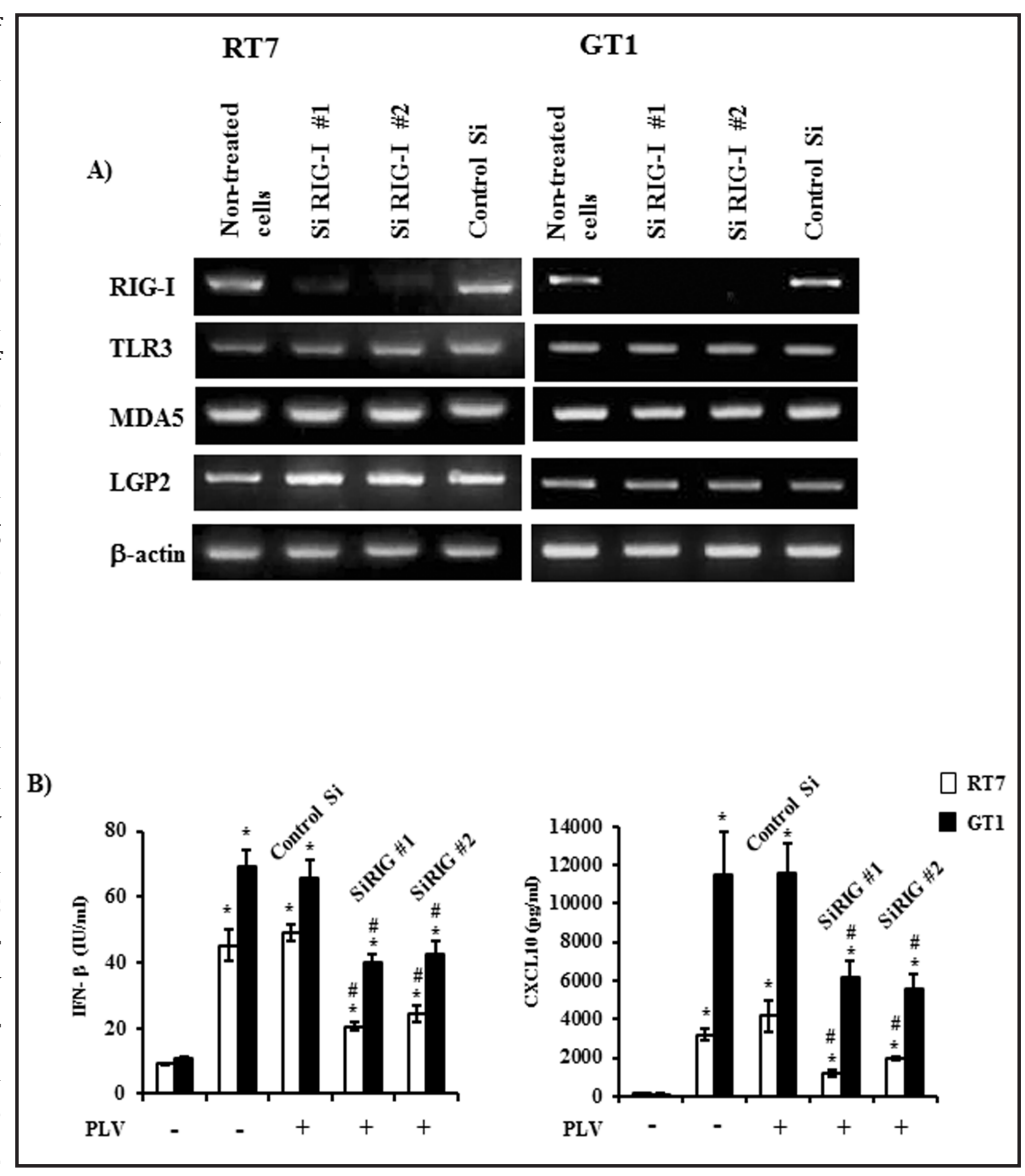
Data are shown as the mean \pm standard deviation of 3 independent experiments. *Significant increase as compared with non-treated cells (Student's $t$-test: $\mathrm{p}<0.05$ ). \#Significant decrease as compared with PLV alone (Student's $t$-test: $\mathrm{p}<0.05$ ).

STAT1 homodimer [20]. Therefore, we examined whether recombinant IFN- $\beta$ activated the production of CXCL10 with STAT1 phosphorylation in RT7 and GT1. Recombinant IFN- $\beta$ induced CXCL10 production in both cells in a dose-dependent manner (Fig. 5A), as well as phosphorylation of STAT1 (Fig. 5B). To confirm the contribution of IFN- $\beta$ to PLV-induced CXCL10 production, neutralizing antibodies to block IFN- $\beta$ signaling were applied. Both the neutralizing antibody to IFN- $\beta$ and the antagonistic antibody to IFN $\alpha / \beta$ receptor (IFNAR) attenuated PLV-induced CXCL10 production in RT7 and GT1 (Fig. 4C).

\section{Discussion}

RIG-I was previously characterized as a cytoplasmic pattern recognizing receptor (PRR) for the viral signature dsRNA that functions in signal type I IFN production against virus infection [4]. In RIG-I ${ }^{-/-}$mice, fibroblasts and dendritic cells failed to generate IFN- $\beta$ upon RNA virus infection [21]. In addition, RIG-I was detected not only in fibroblasts and immune cells, but also in some epithelial cells in the colon, bronchi, and lungs [22-24]. The present study is the first to reveal that oral keratinocytes as well as oral fibroblasts constitutively express functional RIG-I in their cytoplasm.

Synthetic poly I:C/LyoVec, a complex of synthetic poly I:C and the transfection reagent LyoVec functions efficiently as an agonist to cytoplasmic PRRs rather than transmembrane PRR (i.e., TLR3). Among the potent RNA helicase PRRs, RIG-I recognizes dsRNA with a lowmolecular weight (up to $1 \mathrm{~kb}$ ), whereas another intracellular PRR MDA5, which triggers 
Fig. 4. Signal transduction activated by PLV via RIG-I in RT7 and GT1. (A) Effects of PLV on IRF3 and STAT1 phosphorylation. Cells were exposed to PLV (500ng/ $\mathrm{mL}$ ) for the indicated durations. Extracts of cells were subjected to SDS-PAGE and western blotting with antibodies against phospho-specific IRF3, total IRF3, phospho-specific STAT1, and total STAT1. (B) Effects of IRF3 and JAK/ STAT inhibition on PLV-induced IFN- $\beta$ mRNA expression in RT7 and GT1. Cells were pre-incubated with BX795 (1 or $2 \mu \mathrm{M}$ ) or AG490 (1 or $10 \mu \mathrm{M}$ ) for $1 \mathrm{hr}$, then exposed to PLV (500 ng/mL) for 12 hrs. IFN- $\beta$ mRNA levels are shown as relative to $\beta$-actin, used as an internal control, and the values are presented as the mean \pm standard deviation of 3 independent experiments. \#Significant decrease as compared with PLV alone (Student's $t$-test: $\mathrm{p}<0.05$ ). (C) Effects of IRF3 and JAK/STAT inhibition on PLV-induced CXCL10 expression in RT7 and GT1. Cells were preincubated with BX795 (1 or $2 \mu \mathrm{M})$ or AG490 (1 or $10 \mu \mathrm{M})$ for 1 hr, then exposed to PLV (500 ng/mL) for 24 hrs, after which the levels of IFN- $\beta$ and CXCL10 in culture supernatants were determined by ELISA. Data are shown as the mean \pm standard deviation of 3 independent experiments. \# Significant decrease as compared with PLV alone (Student's $t$-test: $p<0.05$ ). (D) Effects of siRNA for RIG-I on PLV-induced STAT1 activation in RT7 and GT1. Cells were transiently transfected with the indicated combinations of the siRNA for RIG-I and the negative control siRNA for $48 \mathrm{hrs}$, then exposed to PLV (500ng/mL) for $6 \mathrm{hrs}$. Extracts of cells were subjected to SDS-PAGE. We examined STAT1 activation by western blotting analysis with antibodies against phospho-specific STAT1, and total STAT1.

mitochondrial IPS1-dependent innate immune signaling response, detects long dsRNA (more than $2 \mathrm{~kb}$ ) [25]. As expected, based on our findings of RIG-I expression in both mRNA and protein of RT7 and GT1, low molecular weight poly I:C/LyoVec dramatically increased the levels of IFN- $\beta$ and CXCL10, while PLV-induced IFN- $\beta$ and CXCL10 levels were decreased 
Fig. 5. Effects of IFN- $\beta$ as autocrine factor on PLVinduced CXCL10 in RT7 and GT1. (A) Effects of IFN- $\beta$ on CXCL10 expression in RT7 and GT1. Cells were exposed to various concentrations of IFN- $\beta$ for 12 hrs, after which the levels of CXCL10 in culture supernatant were measured by ELISA. Data are shown as the mean \pm stand ard deviation of 3 independent experiments. *Significant increase as compared with PLV alone (Student's $t$-test: $p$ $<0.05$ ). (B) Effects of IFN- $\beta$ on STAT1 phosphorylation in RT7 and GT1. Cells

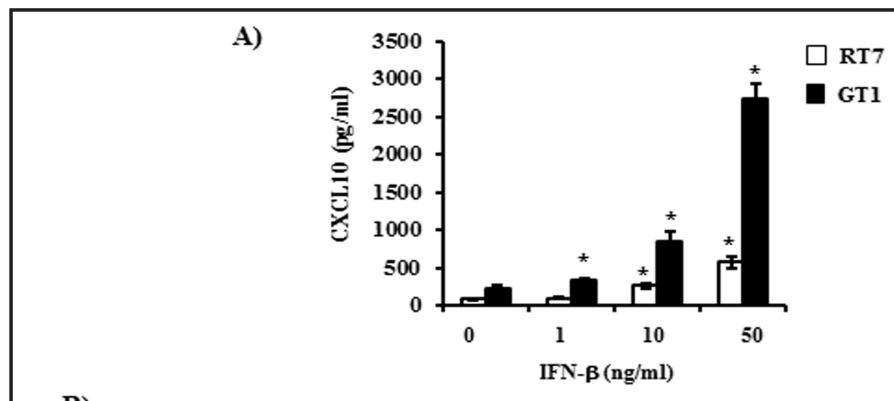

B)

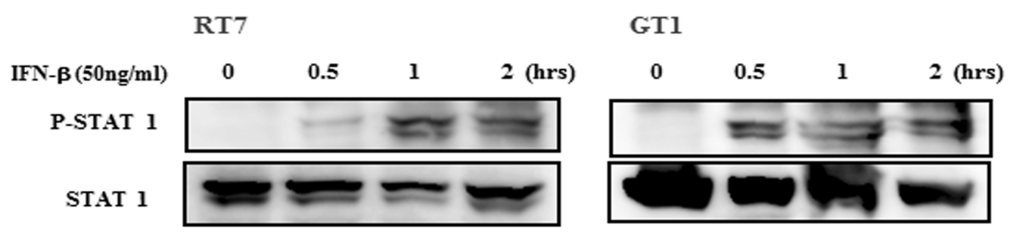

C)
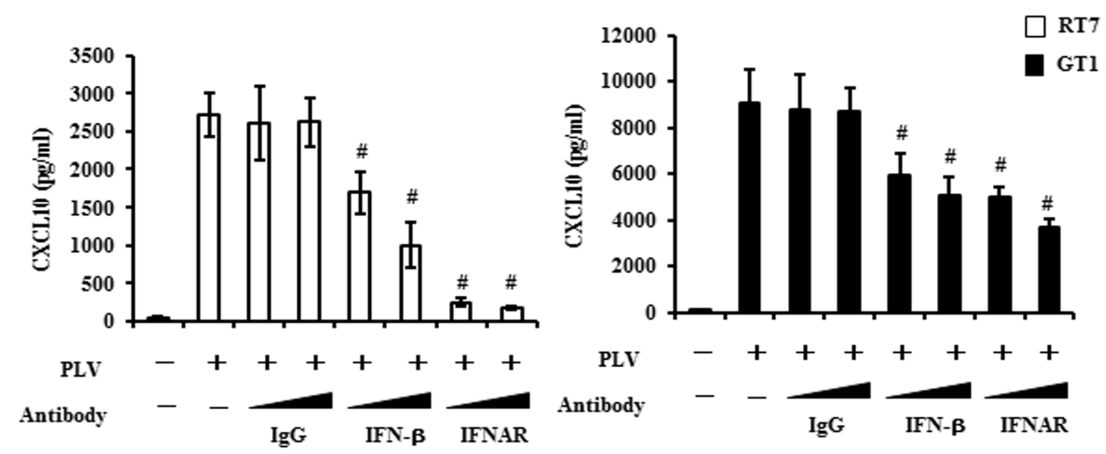

were exposed to PLV (500 ng/mL) for the indicated durations. Cell extracts were subjected to SDS-PAGE and western blotting with antibodies against phospho-specific STAT1, and total STAT1. (C) Effects of blocking antibodies against IFN- $\beta$ and IFN- $\alpha / \beta$ receptor on PLV-induced CXCL10 expression in RT7 and GT1. Cells were pre-incubated with the IFN- $\beta$ antibody, IFN- $\alpha / \beta$ receptor (IFNAR) antibody, and anti-rabbit IgG (10 or $50 \mu \mathrm{g} / \mathrm{mL}$ ) for $1 \mathrm{hr}$, then exposed to PLV (500 ng/mL) for $24 \mathrm{hrs}$, after which the levels of CXCL10 in culture supernatant were measured by ELISA. Data are shown as the mean \pm standard deviation of 3 independent experiments. \# Significant decrease as compared with PLV alone (Student's t-test: $\mathrm{p}<0.05$ ).

by knockdown of RIG-I in those cells. Together, our results suggest that oral keratinocytes and fibroblasts are involved in host defense responses against virus invasion via RIG-I.

RIG-I acts as a major RNA viral sensor in fibroblasts [26]. TLR3 is localized in the endosome membrane, and has been shown to recognize the synthetic dsRNA analogue naked poly I:C. However, TLR3-/- mouse embryonic fibroblasts exhibit normal IFN response with viral infections [20]. MDA5 is a RIG-I-like receptor that senses cytoplasmic viral RNA and induces antiviral responses via the adaptor protein MAVS [25], and MDA5-/- MEFs were shown to produce comparable amounts of IFN- $\beta$ in response to transcribed dsRNAs, whereas RIG-I -/- MEFs did not produce detectable amounts of IFN- $\beta$ in response to those [20]. In the present study, PLV (transcribed Poly I:C/LMW) increased CXCL10 in comparison with Poly I:C/LMW for TLR3 agonists in both types of cells. Especially in RT7, the increase in CXCL10 by PLV was dramatically different from that by Poly I:C/LMW. Furthermore, transfection with siRNA for MDA5 decreased the level of PLV-induced CXCL10 to a much lower level than siRNA for RIG-I in GT1, but not RT7 (data not shown). Although, other extracellular and intracellular PRRs may account for the incomplete suppression of cytokine production in the RIG knockdown experiment (Fig. 3B), intercellular response via RIG-I was shown to have an anti-viral response in oral keratinocyets as well as oral fibroblasts. 
CXCL10 is an inflammatory chemokine that mainly recruits activated T cells and NK cells to sites of infection or inflammation [7]. CXCL10 expression is significantly induced and plays a critical role in host defense with a variety of viral infections and CXCL10-knockout mice have shown a decreased ability to control viral infections, as well as impaired $\mathrm{T}$ cell recruitment and activation [27]. Some investigators have reported that RIG-I is associated with induction of CXCL10 in human bronchial epithelial cells $[17,28]$ and fibroblast-like synoviocytes [29]. In agreement with those findings, we noted that RIG-I in oral keratinocytes and fibroblasts regulated CXCL10 production as an intracellular receptor of dsRNA. On the other hand, it has been reported that RIG-I regulating of CXCL10 is associated with T cell mediated-autoimmune disease, such as rheumatoid arthritis [29]. Anti-virus responses via RIG-I may attract T-cells to the site of infection through CXCL10 to develop T cell-mediated oral inflammation.

STAT1 is a key mediator of cytokine-induced gene expression under type II interferon [20], and activated STAT1 directly regulates the expression of CXCL10 [30]. In the present study, RIG-I-mediated signal transduction resulted in CXCL10 production via the IFNARSTAT1axis in both RT7 and GT1, which was initiated by INF- $\beta$ production in response to PLV exposure, while PLV-induced CXCL10 in RT7 was strongly inhibited by blocking IFNAR and the TBK1 inhibitor in comparison with that in GT1. Therefore, regulation of PLV-induced CXCL10 via RIG-I may be dependent on IRF3/IFN- $\beta$ in oral keratinocytes, while a parallel signaling pathway independent of the RIG-I/IRF3/IFN- $\beta$ axis may exist in oral fibroblasts for CXCL10 production.

In conclusion, this is the first demonstration that 1) Oral keratinocyte as well as oral fibroblast expressed functional RIG-I 2) RIG-I recognized virus mimic ds RNA to produce IFN- $\beta$ and CXCL10 3) RIG-I was essential for activation of IRF3 and STAT1 in response to virus mimic ds RNA 4) RIG-I-IRF3 axis shaped the autocrine IFN- $\beta$-STAT1 axis to produce CXCL10. Taken together, our results indicate that RIG-I in oral keratinocytes and fibroblasts may cumulatively participate in host-defense against viral infection in oral mucosa.

\section{Acknowledgments}

This work was supported by a Grant-in-Aid for scientific research from the Japan Society for Young Scientists (B) from the Ministry of Education, Culture, Sports, Science and Technology (No. 25861946). The authors declare no potential conflicts of interest with respect to the authorship and/or publication of this article.

\section{References}

1 Thompson MR, Kaminski JJ, Kurt-Jones EA, Fitzgerald KA: Pattern recognition receptors and the innate immune response to viral infection. Viruses 2011;3:920-940.

Takeda K, Akira S: Roles of Toll-like receptors in innate immune responses. Genes Cells 2001;6:733-742. Desmet CJ, Ishii KJ: Nucleic acid sensing at the interface between innate and adaptive immunity in vaccination. Nat Rev Immunol 2012;12:479-491.

-4 Yoneyama M, Kikuchi M, Natsukawa T, Shinobu N, Imaizumi T, Miyagishi M, Taira K, Akira S, Fujita T: The RNA helicase RIG-I has an essential function in double-stranded RNA-induced innate antiviral responses. Nat Immunol 2004;5:730-737.

Slots J: Oral viral infections of adults. Periodontol 2000 2009;49:60-86.

Fukui A, Ohta K, Nishi H, Shigeishi H, Tobiume K, Takechi M, Kamata N: Interleukin-8 and CXCL10 expression in oral keratinocytes and fibroblasts via Toll-like receptors. Microbiol Immunol 2013;57:198206.

7 Qin S, Rottman JB, Myers P, Kassam N, Weinblatt M, Loetscher M, Koch AE, Moser B, Mackay CR: The chemokine receptors CXCR3 and CCR5 mark subsets of T cells associated with certain inflammatory reactions. J Clin Invest 1998;101:746-754.

$\checkmark 8$ Ohta K, Shigeishi H, Taki M, Nishi H, Higashikawa K, Takechi M, Kamata N: Regulation of CXCL9/10/11 in oral keratinocytes and fibroblasts. J Dent Res 2008;87:1161-1165. 
9 Klein RS, Lin E, Zhang B, Luster AD, Tollett J, Samuel MA, Engle M, Diamond MS: Neuronal CXCL10 directs CD8+ T-cell recruitment and control of West Nile virus encephalitis. J. Virol 2005;79:11457-11466.

10 Walsh KB, Edwards RA, Romero KM, Kotlajich MV, Stohlman SA, Lane TE: Expression of CXC chemokine ligand 10 from the mouse hepatitis virus genome results in protection from viral-induced neurological and liver disease. J. Immunol 2007;179:1155-1165.

11 Fujimoto R, Kamata N, Yokoyama K, Taki M, Tomonari M, Tsutsumi S, Yamanouchi H, Tomonari M, Nagayama M: Establishment of immortalized human oral keratinocytes by gene transfer of a telomerase component. J Jpn Oral Muco Membr 2002;8:1-8.

12 Kamata N, Fujimoto R, Tomonari M, Taki M, Nagayama M, Yasumoto S: Immortalization of human dental papilla, dental pulp, periodontal ligament cells and gingival fibroblasts by telomerase reverse transcriptase. J Oral Pathol Med 2004;33:417-423.

13 Spivack SD, Hurteau GJ, Jain R, Kumar SV, Aldous KM, Gierthy JF, Kaminsky LS:Gene-Environment Interaction Signatures by Quantitative mRNA Profiling in Exfoliated Buccal Mucosal Cells. Cancer Res 2004;64:6805-6813.

14 Gitlin L, Barchet W, Gilfillan S, Cella M, Beutler B, Flavell RA, Diamond MS, Colonna M: Essential role of mda5 in type I IFN responses to polyriboinosinic:polyribocytidylic acid and encephalomyocarditis picornavirus. Proc Natl Acad Sci U S A 2006;103:8459-8464.

15 Hayes TG : Differences between human alpha (leukocyte) and beta (fibroblast) interferons. Arch Virol 1981;67:267-281.

-16 Fensterl V, Grotheer D, Berk I, Schlemminger S, Vallbracht A, Dotzauer A: Hepatitis A virus suppresses RIGI-mediated IRF-3 activation to block induction of beta interferon. Virol 2005;79:10968-10977.

17 Imaizumi T, Kumagai M, Taima K, Fujita T, Yoshida H, Satoh K: Involvement of retinoic acid-inducible gene-I in the IFN- $\gamma$ /STAT1 signaling pathway in BEAS-2B cells. Eur Respir J 2005;25:1077-1083.

18 Chariot A, Leonardi A, Muller J, Bonif M, Brown K, Siebenlist U: Association of the adaptor TANK with the I kappa B kinase (IKK) regulator NEMO connects IKK complexes with IKKe and TBK1 kinases. J. Biol. Chem 2002;277:37029-37036.

19 Clark K, Plater L, Peggie M, Cohen P: Use of the pharmacological inhibitor BX795 to study the regulation and physiological roles of TBK1 and IkappaB kinase epsilon: a distinct upstream kinase mediates Ser-172 phosphorylation and activation. Biol Chem 2009;284:14136-14146.

20 Gough DJ, Messina NL, Hii L, Gould JA, Sabapathy K, Robertson AP, Trapani JA, Levy DE, Hertzog PJ, Clarke CJ, Johnstone RW: Functional crosstalk between type I and II interferon through the regulated expression of STAT1. PLoS Biol 2010;2: DOI: 10.1371/journal.pbio.1000361.

21 Kato H, Sato S, Yoneyama M, Yamamoto M, Uematsu S, Matsui K, Tsujimura T, Takeda K, Fujita T, Takeuchi O, Akira S: Cell type-specific involvement of RIG-I in antiviral response. Curr Opin Immunol 2010;22:41-47.

22 Mukherjee A, Morosky SA, Shen L, Weber CR, Turner JR, Kim KS, Wang T, Coyne CB: Retinoic acid-induced gene-1 (RIG-I) associates with the actin cytoskeleton via caspase activation and recruitment domaindependent interactions. J Biol Chem 2009;6:6486-6494.

-23 Slater L, Bartlett NW, Haas JJ, Zhu J, Message SD, Walton RP, Sykes A, Dahdaleh S, Clarke DL, Belvisi MG, Kon OM, Fujita T, Jeffery PK, Johnston SL, Edwards MR: Co-ordinated role of TLR3, RIG-I and MDA5 in the innate response to rhinovirus in bronchial epithelium. PLoS Pathog 2010;6:DOI: 10.1371/journal.ppat.1001178.

-24 Pothlichet J, Meunier I, Davis BK, Ting JP, Skamene E, von Messling V, Vidal SM: Type I IFN triggers RIG-I/ TLR3/NLRP3-dependent inflammasome activation in influenza A virus infected cells. PLoS Pathog 2013;9:DOI: 10.1371/journal.ppat.1003256.

25 Wilkins C, Gale M Jr: Recognition of viruses by cytoplasmic sensors. Curr Opin Immunol 2010;22:41-47.

26 Yoneyama M, Fujita T: Function of RIG-I-like receptors in antiviral innate immunity. J Biol Chem 2007;282:15315-15318.

27 Dufour JH, Dziejman M, Liu MT, Leung JH, Lane TE, Luster AD: IFN- $\gamma$-inducible protein 10 (IP-10; CXCL10)-deficient mice reveal a role for IP-10 in effector T cell generation and trafficking. J. Immunol 2002;168:3195-3204.

28 Taima K, Imaizumi T, Yamashita K, Ishikawa A, Fujita T, Yoshida H, Takanashi S, Okumura K, Satoh K: Expression of IP-10/CXCL10 is upregulated by double-stranded RNA in BEAS-2B bronchial epithelial cells. Respiration 2006;73:360-364.

29 Imaizumi T, Arikawa T, Sato T, Uesato R, Matsumiya T, Yoshida H, Ueno M, Yamasaki S, Nakajima T, Hirashima M, Sakata K, Ishibashi Y, Toh S, Ohyama C, Satoh K: Involvement of retinoic acid-inducible gene-I in inflammation of rheumatoid fibroblast-like synoviocytes. Clin Exp Immunol 2008;153:240-244.

-30 Wong LH, Sim H, Chatterjee-Kishore M, Hatzinisiriou I, Devenish RJ, Stark G, Ralph SJ: Isolation and characterization of a human STAT1 gene regulatory element: inducibility by interferon (IFN) type I and II and role of IFN regulatory factor-1. J Biol Chem 2002;277:19408-19417. 